DWBA to coupled channel problems. As an alternative theoretical framework, Levin ends with a description of the much more controversial stripping theory of Butler and co-workers, comparing it with both the DWBA and experiment.

The article of Feshbach is very different in character. In just 45 pages it describes some of the features of the theory of nuclear reactions which the author and his collaborators at MIT have developed over the years. Central to this is the interplay between the prompt reactions (the direct reactions discussed by Levin) and the timedelayed ones involving the formation of compound nuclei. By the elimination of the time-delayed components, he shows how an optical model description of the prompt reactions can be formulated, stressing the importance of energy averaging in the scheme. This division into prompt and delayed reactions is of course oversimplistic and Feshbach finishes by considering reactions asociated with characteristic times intermediate between these two extremes, such as doorway states. Unlike the Levin work, these feel much more like lecture notes, intended to give the student a broad overall impression of the field. Colin WILKIN

\section{Light on molecules}

Organic Molecular Photophysics. Vol. 1. Edited by John B. Birks. Pp. xviit 600 (Wiley Monographs in Chemical Physics.) (Wiley-Interscience: London and New York, July 1973.) £13.50.

THIs book is the first of two volumes aiming to provide "comprehensive coverage of the field of organic molecular photophysics" and is intended to be complementary to Photophysics of Aromatic Molecules written by Birks. This volume contains ten articles, ranging in subject content from organic dye lasers to exciton interactions in organic solids, which have been written, on the whole, by unquestionable experts in their fields.

The quality of individual articles is extremely high. For example, the treatment of radiationless transitions by Henry and Siebrand presents one of the clearest discussions on this complicated topic. The fluorescence characteristics of aromatic molecules is dealt with thoroughly in two chapters, one by Stockburger, considering low pressure environments and the other, by Offen, dealing with fluorescence and absorption effects at high presures. Triplettriplet absorptions are discussed from an experimental standpoint by Labhart and Heinzelmann who compile much of the data available up to 1971. There is perhaps some overlap in tabular content between this chapter and "Photo- physics of Aromatic Molecules". Dye lasers are dealt with from an experimental and occasional mathematical approach by Snavely; it is a pity that there is little mention in this article on their obvious uses in, for example, high resolution spectroscopy or fluorescence studies of vibronic states. Klopffner deals thoroughly with intermolecular exciplexes. The first chapter on the spectroscopy of pi-electronic states (by Birks) is introductory in nature and those familiar with the author's first work in this series will find the chapter less useful than the rest of the book. Other articles are written on diffusioncontrolled rate processes (Alwattar, Lumb and Birks), electron photoejection from aromatic molecules in condensed media (Lesclaux and JoussotDibien) and exciton interactions in organic solids (Swenberg and Gaecintov).

I found it difficult to regard the collection of articles as "integrated" since the book reads more like a high quality "Advances in . . ." series. It could be argued that most of the review articles are available elsewhere although not in a single volume. I hope that the high standard of individual articles is maintained in volume 2 . This volume would be a very useful addition to the library of individual researchers engaged in photochemistry and photophysics although it would not be essential.

$$
\text { M. A. WEST }
$$

\section{Atmospheric chemistry}

Chemistry of the Lower Atmosphere. Edited by S. I. Rasool. Pp. xii +335 . (Plenum: New York and London, 1973.) $\$ 26$.

THE book is a collection of six chapters on various aspects of atmospheric physics and chemistry by six different authors. Like all such collections it is variable in emphasis, standard and depth of treatment of subject. Because of this it is difficult to decide to what audience the book is aimed.

Dealing with each chapter in turn, the first of about 60 pages entitled "Role of Natural and Anthropogenic Pollutants in Cloud and Precipitation Formation", by Professor H. R. Pruppacher of UCLA, does no more than summarise what can be found in the standard texts on cloud microphysics. Although lip service is paid to the importance of cloud dynamics, that is the air motions leading to cloud condensation, and the interaction between cloud microphysics and dynamics, in fact the chapter is almost exclusively devoted to the microphysics of clouds. There is nothing on the possible modification of precipitation due to anthropogenic pollutants except a brief uncritical reference to some evidence that urban areas experience more precipitation than their adjoining rural environment.

The next chapter, of about 40 pages on "Particulate Matter in the Lower Atmosphere" by Dr R. D. Cadle of NCAR, is a standard review of the main physical and chemical properties of the tropospheric and stratospheric aerosols. The sections on aspects of the atmospheric sulphur cycle and the scavenging processes for particulates in the troposphere duplicate more extensive treatments of these aspects in later chapters.

The third chapter is on "Removal Processes of Gaseous and Particulate Pollutants" by Dr G. M. Hidy, and is perhaps the best chapter in the book. It contains a scholarly and comprehensive treatment of the atmospheric scavenging processes.

"The Global Sulphur Cycle" is the title of the next section (24 pages) by Dr J. P. Friend. It summarises effectively the current state of knowledge of sources, sinks and reservoirs of sulphur. It is nice to see an author emphasising the limitations of his treatment.

The next contribution of 46 pages is perhaps the one closest to the central theme of the book, in that it deals with "The Chemical basis for Climate Change". The authors are Drs Schneider and Kellogg of NCAR. In it are described the qualitative arguments used to ascribe possible casual relationships between changes in atmospheric composition and climate. It also gives the reader some feel for the vast problems involved in any worthwhile quantitative modelling of climatic change.

The last chapter of 79 pages, by $\mathrm{Dr}$ C. D. Keeling, is devoted to the carbon dioxide cycle. It could be argued that the mathematical detail contained in this chapter is out of proportion to that in the rest of the book, but nevertheless it gives a very real insight into the various interacting factors concerned with the atmosphere, the oceans and the biosphere which control the concentration of $\mathrm{CO}_{2}$ in our atmosphere.

In summary, the book has little to offer those workers actively engaged in the fields of atmospheric chemistry and meteorology. But at a time when more and more scientists outside the fields of atmospheric physics and chemistry are taking an interest in pollution matters, this book provides a useful introduction to some aspects of the subject.

P. GoldsMith

\section{Erratum}

The price given for Comparative Vertebrate Morphology by Douglas and Molly Webster (reviewed in Nature, 249, 866; 1974) was incorrect; it should have been $\$ 14.95$ and $£ 7$. 